\title{
Kickstarting the Endoscopy Unit after Lockdown Period: Why, When, and How?
}

\author{
Zaheer Nabi ${ }^{1} \quad$ D. Nageshwar Reddy ${ }^{1}$ \\ ${ }^{1}$ Department of Gastroenterology, Asian Institute of \\ Gastroenterology, Hyderabad, Telangana, India
}

J Digest Endosc 2020;11:92-93

Endoscopy is an aerosol-generating procedure which poses a high risk of transmission to the endoscopist. Consequently, only urgent and semiurgent endoscopic procedures are being performed since the dawn of the lockdown period. Curbing down the number is the need of the hour and not the choice. It is meant to prepare us to embrace the uncertain conditions in the postlockdown era. In addition, it would allow the judicious use of health care resources like personal protection equipment (PPE) and save them for a time when they are needed the most.

In the article, "Way forward: GI Endoscopy Work flow post Lockdown era," the authors provide with a broad guidance on the strategies of stepwise resumption of endoscopy services in the postlockdown phase.

\section{Preparations during the Lockdown Period: "You reap what you sow"}

The lockdown phase has provided us with an opportunity to prepare and deal with the nuances associated with endoscopies during COVID-19 era. In this period, the gastrointestinal (GI) endoscopy unit should intensify the training of the endoscopist as well as the assistants in methods to prevent transmission of infection to the health care professionals (HCPs). It may not be easy to change practices which have been hardwired over many years. The endoscopy suit should be prepared in such a way that there are minimum extra surfaces (like tables, chairs, writing pads) which may act as potential sources of transmission. In addition, a separate corridor should be created for the transfer of high-risk versus low-risk patients.

\section{Endoscopies in the Near Future: The Balancing Act}

It is likely that the volume of endoscopies in the near future will not be the same as the prepandemic phase. Apprehensions would linger on with the endoscopists as well as the patients scheduled for endoscopies in the
Address for correspondence D. Nageshwar Reddy, MD, DM, Asian Institute of Gastroenterology, Hyderabad 500082, Telangana, India (e-mail: aigindia@yahoo.co.in).

postlockdown phase. The approach in the postlockdown phase needs to be a balanced one. A lot of endoscopies would have been cancelled or rescheduled by the time the lockdown phase is over. Consequently, the toll on non-COVID patients may be much higher than presumed. ${ }^{1}$ The benefit of further delaying these procedures should be weighed and balanced against the risks of denying them repeatedly. Sud et $\mathrm{al}^{2}$ rightly pointed out in this article "Way forward: GI Endoscopy Work flow post Lockdown era" that nonurgent endoscopies cannot be delayed forever and we need to have a blueprint to deal with this situation. The question is "How to go about it"? Well, there is no full proof plan or time-tested strategy here. Uncertainties regarding the duration of lockdown as well as the availability of PPE are major hurdles in the planning a strategic increase in the workflow in GI endoscopy units. The societal guidelines from various GI societies like the Society of Gastrointestinal Endoscopy of India, American Society for Gastrointestinal Endoscopy, Asian Pacific Society for Digestive Endoscopy, and European Society of Gastrointestinal Endoscopy provide guidance on the strategies and precautions required while performing endoscopies during these times. ${ }^{3-6}$ To be precise, these are position statements (not guidelines) stressing the fact that our knowledge on this subject is still evolving and these statements are likely to be updated as we get more evidence.

\section{Regaining Normalcy in GI Unit: "One Size does not Fit All"}

The authors aptly mention that the first and the foremost step prior to scheduling an endoscopy is screening of patients and stratifying them into different risk groups according to symptoms, contact, and travel history. It is important to note that presymptomatic CoV-2 infections are not uncommon and constitute the major hurdle for the current screening strategies. ${ }^{7}$ In addition, the social stigma associated with COVID-19 in our country may pose a special challenge in getting an accurate contact or travel history. Therefore, it may be 
worth rescheduling the endoscopy after 2 to 3 weeks in case a reliable history cannot be obtained.

In this manuscript, the authors suggested that "We should plan to run our facilities at 50\% capacity for next 3 months and change it afterwards according to prevailing situation." However, the strategies to resume normalcy in the postlockdown phase should be individualized in each center. For example, different GI units may not be equal with respect to the age and comorbidity status of HCP, volume of cases, number of health care staff, availability of PPE, and other measures to prevent person-to-person transmission. Some units are known to perform complex and time-consuming therapeutic endoscopic procedures like endoscopic mucosal resections, endoscopic submucosal dissections, therapeutic endosonography procedures, peroral endoscopic myotomy, etc. These procedures by virtue are prone to generate more aerosols than short endoscopic procedures and may pose a higher risk to HCP than short endoscopy procedures. Consequently, the GI units need to devise their own strategies in the postlockdown phase and the "50\%" rule may not fit to all.

\section{Conclusion}

In this challenging time, a clear vision is required to allow organization and smooth transition of the endoscopy services to the "new normal" after the lockdown phase is over. The path to normalcy in GI units does not appear to be a straight one and resurgence in COVID-19 cases after the lockdown phase may pose new challenges in this regard. We hope for the best, but need to be prepared for the worst.

\section{Financial Disclosure}

None.

\section{Conflict of Interest}

None declared.

\section{References}

1 Rosenbaum L. The untold toll - the pandemic's effects on patients without COVID-19. N Engl J Med 2020;10.1056/ NEJMms2009984

2 Sud R, Sud S. Way forward : GI Endoscopy Work flow post Lockdown era. Journal of Digestive Endoscopy 2020;11;89-91

3 Philip M, Lakhtakia S, Aggarwal R, et al. Joint guidance from SGEI, ISG and INASL for gastroenterologists and gastrointestinal endoscopists on the prevention, care, and management of patients with COVID-19. J Clin Exp Hepatol 2020 (epub ahead of print), DOI:10.1055/s-0040-1709837

4 Chiu PWY, Ng SC, Inoue H, et al. Practice of endoscopy during COVID-19 pandemic: position statements of the Asian Pacific Society for Digestive Endoscopy (APSDE-COVID statements) Gut 2020;:gutjnl-2020-321185

5 Gralnek IM, Hassan C, Beilenhoff U, et al. ESGE and ESGENA Position Statement on gastrointestinal endoscopy and the COVID-19 pandemic. Endoscopy 2020;/a-1155-6229

6 Repici A, Maselli R, Colombo M, et al. Coronavirus (COVID-19) outbreak: what the department of endoscopy should know. Gastrointest Endosc 2020;pii:S0016-5107(20)30245-5

7 Arons MM, Hatfield KM, Reddy SC, et al. Presymptomatic SARS-CoV-2 infections and transmission in a skilled nursing facility. N Engl J Med 2020;/NEJMoa2008457 
